\title{
Application of Big Data Analysis Method in Supply Chain
}

\author{
Dou Xin-xin, Wang Xiao-ping \\ School of Logistics, Beijing Wuzi University, Beijing, China \\ Email address: \\ 1185313260@qq.com (Dou Xin-xin),bjwxp2004@163.com (Wang Xiao-ping)
}

To cite this article:

Dou Xin-xin, Wang Xiao-ping. Application of Big Data Analysis Method in Supply Chain. Advances in Networks.

Vol. 4, No. 1, 2016, pp. 1-5. doi: 10.11648/j.net.20160401.11

Received: August 21, 2016; Accepted: August 29, 2016; Published: August 30, 2016

\begin{abstract}
Big data has the characteristics of large scale, many kinds, fast generation, high value but low density. Big data application is the use of data analysis methods, from the big data mining effective information, to provide users with auxiliary decision-making, to realize the process of large data value. In the era of limited information processing capacity, the world needs data analysis, but the lack of tools used to analyze the collected data, so the big data analysis method came into being. Big data analysis has changed a lot of business areas, especially in the supply chain management of the biggest change in the application. This paper mainly introduces the application of big data analysis method and the application in the supply chain, and further expounds the future development of big data analysis method in the future.
\end{abstract}

Keywords: Big Data, Big Data Analysis Method, Supply Chain Management

\section{Introduction}

Over the past 20 years, there have been massive data growth in all areas, including health care and scientific sensors, user generated data, Internet and financial companies, supply chain systems, and so on. In 2011 the world was created and copied the total amount of data for $1.8 \mathrm{ZB}$, an increase of nearly 9 times in just 5 years, and this figure is expected to double at least once every two years. The term big data is generated in the context of the growth of the global data explosion, used to describe a large collection of data. Compared with the traditional data collection, large data usually contains a large number of unstructured data, and large data needs more real-time analysis. In addition, the big data also brings new opportunities for mining hidden value, while giving us a new challenge, that is, how to effectively organize and manage these data. Today, the industry, the research community and even government departments have a huge interest in the research area of big data.

With the development of information technology and the increase of industrial uncertainty, the relationship between enterprises is becoming more and more obvious. At the same time, people's understanding of the supply chain is from the linear single stranded steering nonlinear chain network, the concept of supply chain pay more attention to around the enterprise core network chain, core enterprise and suppliers, supplier of all forward relationship, and the user, the user and all backwards. Supply chain concept is different from the traditional sales chain, it spans the enterprise boundaries, starting from the extended enterprise new thinking, and from the overall situation and the overall point of view considering the competitiveness of products, rise from an operational tool for a kind of management system in the supply chain, a kind of operation management and mode of thinking.

In the era of big data, the data was the world. Will big data is applied to the supply chain has become the competition among enterprises is the key, enterprises through the supply chain collaborative management and planning can reduce the bullwhip effect in the supply chain, so as to promote the supply chain each link run smoothly, which will give rise to intelligent supply chain. This is also the research point of this paper.

\section{Overview of Big Data}

\subsection{Big Data Definition}

In the DT era, the role played by the big data has been self-evident, but the definition of big data but different opinions. Big data is an abstract concept, the removal of a large amount of data, big data as well as some of the other features, these features determines the difference between the concepts of big data and "massive data" and "very large data. 
Generally, big data is the data set that can not be perceived, acquired, managed, processed and served by the traditional IT technology and the software and hardware tools in a limited time. Science and technology enterprises, research scholars, data analysts and technical consultants, due to their different concerns, the big data has a different definition. Through the following definitions, perhaps we can help us better understand the profound meaning of big data in social, economic and technical and other parties, and [1].

Big data is currently the mainstream of large data is defined as $3 \mathrm{~V}$, that is, scale (Volume), diversity (Variety) and high speed (Velocity). The so-called scale is the amount of data reached a certain height, not through the current mainstream tools to timely treatment; diversity that is for the type of data is to deal with, in addition to a structured and semi-structured and unstructured, an increase of the complexity of the operation; high speed is refers to the data arrival and must deal with in a timely and efficient manner, does not allow a longer delay. In addition, it is generally believed that privacy and the value of the same data is the main feature of the main features of [2].

\subsection{Big Data Analysis Method}

With the arrival of the era of big data, how to quickly extract the key information from these massive data, to bring value to businesses and individuals, is the focus of attention of all sectors. At present, some large data processing methods are mainly:

1) Bloom Filter: bloom filter, which is essentially a bit array and a series of Hash functions. The bloom filter principle is using digit set of stored data of the hash value and not the data itself, its essence is lossy compression storage of the bitmap index on the data using the hash function. The advantage of the utility model is that the utility model has the advantages of high spatial efficiency and querying speed, and the disadvantage is that the recognition rate and the deletion difficulty are certain. Bloom filter suitable for large data applications allow low false recognition rate.

2) Hashing: hash method, also called the Hash method, is the essence of the data into the length of the shorter length of the numerical or index value method. The advantage of this approach is that it has fast reading and writing and querying speed, the disadvantage is that it is difficult to find a good Hash function.

3) index: both in the structured data management of traditional relational databases and management technology of semi structured and unstructured data index is a reduce disk read and write an effective method for overhead and improve the additions and deletions to change search rate. The index of the defect is the need for additional overhead storage index files, and need to be updated according to the data and dynamic maintenance.

4) Trie tree: also known as dictionary tree, Hash tree is a variant form, is widely used for fast retrieval, and word frequency statistics. Trie tree's idea is to use the string of common prefix, to maximize the reduction of string comparison, improve query efficiency.

5) parallel computing: compared to the traditional serial computing, parallel computing refers to the use of multiple computing resources at the same time. The basic idea is to decompose the problem, by a number of independent processors to complete their tasks, in order to achieve the purpose of cooperative processing. At present, the typical parallel computing model has MPI (passing interface message), MapReduce, Dryad and so on [3].

Traditional data analysis methods, most of them are sampling or filtered through the original data set, then the sample data analysis, find the characteristic and regularity, its biggest characteristic is through a complex algorithm from a finite sample space to obtain information as much as possible. With the computational capability and memory, data analysis method and the traditional analysis methods, the biggest difference lies in the analysis of all data, and not sample data, its biggest characteristic is not the pursuit of complexity and accuracy of the algorithm, and the pursuit of the analysis can be efficiently on the entire data set. In short, traditional data and methods strive to through complex algorithm from limited data centralized access to information, the more the pursuit of accuracy; data analysis method is through efficient algorithm, pattern, of all the data were analysis [4].

\section{Supply Chain Overview}

\subsection{Definition of Supply Chain Management}

The concept of supply chain is from the expansion of production (extended popularizes concept development, the modern education management of the supply chain the definition of "supply chain is around the core enterprise, through the control of commodity flow, information flow, logistics, capital flow, from the beginning of the procurement of raw materials, intermediate products and final products, and finally by the sales network products to the consumers in the hands of the suppliers, manufacturers, distributors, retailers, until the end user connected into a whole function nets chain structure. Users connect into a whole function network chain structure. It covers all join in enterprise, not only is a connection of all participants of logistics industry chain material, capital chain, information chain or a value-added chain, let the material in the supply chain through processing, transport and packaging process of appreciation, bring benefits to the enterprise.

Supply chain management is a kind of integrated management thought, which performs in the supply chain of logistics planning and control functions, is the process of planning and control of the supply chain logistics, information flow and capital flow, business flow, value flow and between the trading partner relationship. Supply chain management includes purchasing, manufacturing, sales, warehouse management, transportation, storage and customer service. The characteristics of the supply chain management is to focus on the relationship between the level of customer service and the total logistics cost and to maximize the supply chain management efficiency as a whole play out, to achieve the purpose of the enterprise supply chain all benefit. 


\subsection{Supply Chain Link}

The supply chain consists of 4 parts: purchasing, manufacturing, logistics and sales. In the industry leading enterprises are well aware that only focus on the part of a is not enough, if any part of the information cannot be passed on to other departments will likely will lead to purchase link shortage and lag of logistics, manufacturing sectors appear the stagnation of production conditions. The four part of the presentation is as follows:

1) Procurement. procurement mainly refers to the supply or purchase, refers to all the activities and processes responsible for the purchase of goods and services from suppliers. Supply of goods has an important economic impact on the organization. Enterprises spend a lot of money on procurement, the process of rational planning can save money, which has a significant meaning to the enterprise. In most manufacturing enterprises, the procurement process represents the company's largest expenditure, will account for the total cost of $50 \% \sim 90 \%$ [5].

2) Manufacturing refers to the operation of an organization that is responsible for creating products and services. Manufacturing plays a role of transportation in the enterprise operation, and the investment of the enterprise is converted into the final product. These inputs include raw materials, science and technology, information, human resources and technical facilities. Manufacturing output is the product and service of enterprise production, which covers medical services, toys and clothing and so on. [6].

3) Logistics. logistics refers to the business in the supply chain, which will deliver the products to the designated place within the specified time. Logistics enables the production of products in the supply chain to flow and storage, and related decisions including the calculation of inventory, coordination and allocation of raw materials, arrangements for the distribution route as well as freight [7].

4) Sales in the supply chain is the marketing, it will be linked to the enterprise and consumers to determine the consumer demand, promote new product consumption and found that market opportunities. An organization and its supply chain want to remain competitive, it is necessary to better meet consumer demand than competitors, this is the role of sales links to play.

5) Supply chain integrated application of large data analysis can achieve intelligent supply chain. Below will introduce some of the forefront of the application of information technology supply chain, they go beyond the traditional marketing role, and is used to record the management of inventory movement, create a sales and supply chain management information feedback mechanism.

\section{Big Data Promote the Supply Chain Transformation}

\subsection{The Impact of Big Data Analysis on Purchasing}

In recent years, more and more retail companies use big data technology to optimize its procurement process, change its procurement business model, and the results are significant. Future retail enterprises in the business model transformation process will be due to the impact of large data caused profound changes in the retail sector purchasing business process, the new procurement business processes will be able to for enterprises to reduce procurement costs, reduce procurement links, improve procurement efficiency, can provide more accurate prediction of procurement information, avoid the procurement of the black hole to purchase more transparent and more standardized.

First, the traditional procurement model of the procurement business processes as shown in Figure 1 below [8]. The main disadvantages of the process are: the lack of necessary supervision and control mechanisms; it is easy to bring unnecessary inventory backlog and increase the amount of accounts payable. Secondly, under the traditional procurement mode, business information sharing is weak, the business can be traced back to the weak, the problem is difficult to investigate; personnel changes in the business of great impact on the business. Finally, under the traditional procurement mode, the control of the procurement process is often after the control, it will cause unnecessary losses to the enterprise.

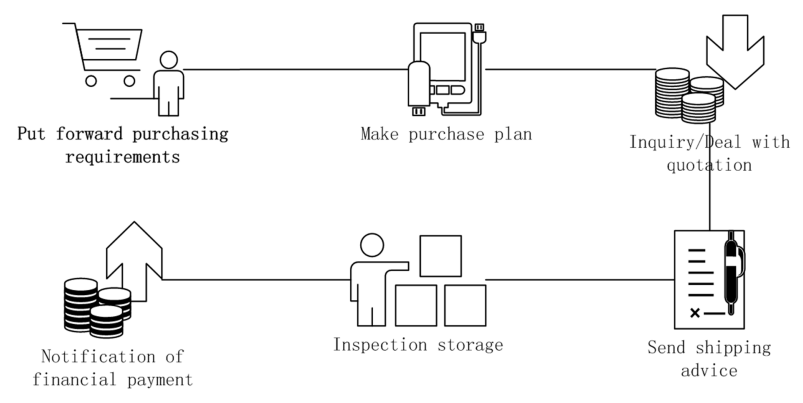

Figure 1. Procurement business process under the traditional model.

Big data to drive the transformation of business model, so that the procurement of business activities into electronic procurement. And e-procurement has the following advantages: first, purchasing information comprehensive, accurate convenient management decision-making; second, procurement process is fair and equitable, improve the transparency of procurement; third, streamlining the procurement process, saving time cost.

To sum up, the impact of large data analysis for the procurement process is very important. Enterprises can use big data to optimize the procurement channel selection, the supplier integrated into the data system. Some companies will also use big data to identify the characteristics of each supplier, to provide information for negotiations with suppliers. Big data analysis can also analyze the customer's preferences and buying behavior, which in turn can provide information for enterprises to negotiate with suppliers. Companies can use these prices and trading information for their own franchise rights for key products.

\subsection{Effect of Large Data Analysis Method on Manufacturing}

Enterprises are widely used in this part of the data analysis 
to improve the production of all aspects of. Many enterprises will be the big data analysis in the application of inventory management, optimize the inventory level, optimize the maintenance of the location and other aspects of the update and update the equipment. In the productivity and quality, big data analysis has broad application space. Enterprise can analyze the daily production performance, its data source store sales, inventory unit sales and unit staff sales. At present, these systems are more and more close to real-time reporting, as well as real-time to remind the enterprise productivity changes or decline in quality and other issues. Enterprises can view the production accuracy and product quality by observing labor data. Today, the trend of the use of large data analysis is high frequency, fast and granular data reports, to help enterprises in a timely manner to make targeted adjustments.

Labor force optimization is another application of big data analysis. This technology through the optimization of labor, automated tracking investigation, improve labor schedule, while maintaining the service level and reduce production costs. For example, call center supervisor can analyze customer service quality according to customer complaint and satisfaction survey. Data analysis is also able to better predict human needs, and the results of the forecast and the optimization of the labor force, which is particularly important in the peak of human demand.

\subsection{The Impact of Big Data Analysis on Logistics}

Big data analysis in this area is applied in the optimization of storage, replenishment inventory, distribution center optimization location and transportation costs to minimize these aspects. Data analysis can be customer groups, companies can be divided into groups to divide traffic routes, traffic patterns and the delivery of different products of the relevant elements. Data analysis is the most widely used in the field of transport and route choice. Company can use the loading GPS navigation data remote information processing technology and route optimization to improve freight traffic, traffic analysis can improve fuel efficiency, preventive maintenance, driver behavior and vehicle route optimization, so as to improve the productivity level [9].

Another big data application area is inventory management. Radio frequency identification technology in this area has a significant role, it can be applied in the dynamic tracking inventory, determine the location and quantity of goods and to avoid the risk of security risks in these areas. Radio frequency identification is also able to detect the temperature around the route, to ensure food safety, planning and delivery of shelf life and timely notification to the decision maker.

\subsection{The Impact of Big Data Analysis on Sales}

Market segment, forecast consumer behavior is an important application of large data analysis, companies can collect and track the individual consumer behavior data, combined with traditional marketing tools. Designed specifically for the study of consumer behavior and the design of the application towards the direction of precision development, making big data in the field of sales has experienced an unprecedented growth. Enterprises in this field for large data analysis is far more than the application of customer and market division, but the use of it on consumer behavior from the localization of marketing. Emotional analysis to the store's consumer behavior in all aspects of interpretation and tracking.

\section{The Case Analysis}

WAL-MART is a typical example of the application of smart supply chain [10]. WAL-MART has established a new supply chain system, in series with the vast majority of supply chain decision-making, and its optimization. This system belongs to the "non stability stochastic model", which is to fulfill the order, supply of goods, operational capacity and inventory. WAL-MART established a new algorithm for joint replenishment inventory, in the implementation of the new unit level prediction method. The model is based on historical demand, taking into account historical events and future planning, forecasting the performance of each order, inventory planning, purchasing cycle and purchase order. In essence, the model is connected in series with the whole supply chain, which can collect a large number of sales and inventory data, because WAL-MART has more than 1 million customers per hour. All data will be entered into the integrated technology platform for processing. Here, the company managers take turns to analyze various aspects of the supply chain, to make a comprehensive coordination, based on the decision of the data.

WAL-MART collects a large number of customer data, it is stored in the database. WAL-MART has the amount of data in fact has exceeded any one of the private sector has the amount of data, the Wal-Mart Store Inc is based on this huge database of tens of thousands of marketing analysis. Any organization on the WAL-MART supply chain can be used for data. The warehouse manager uses the system to analyze the specific sales data, optimize the product classification, and distribute the products to the local community through the quality inspection. WAL-MART's marketing staff to determine customer needs through data, as well as the time and place of demand. Through the analysis of the data, the understanding of the company to before the hurricane will be stored without cooking or refrigerated storage of food, such as Kellogg's tart biscuits, especially strawberry tart biscuits. WAL-MART and Kellogg cooperation, to step up the purchase, in order to prepare for the need for hurricanes. Is the customer shopping nuanced observation enables Wal Mart to more deeply understand customer preferences and shopping behavior, also let Wal Mart from suppliers get the pricing and distribution franchise.

Wal Mart's analysis and data on the distribution of more than 17400 vendors to open in 80 countries, each vendor can track their products through the company's retail chain platform, which can make the demand of supply chain understanding market different products in, real-time understanding of business needs to purchase time, rather than passively waiting orders of Wal Mart. In addition suppliers 
were able to database information query, search sales and shipments, orders, invoices, claims and notice and other relevant information, you can enter the Wal Mart products classification scheme of the system, in the sales data and store characteristics based on created specifically for the store to create modular layouts.

WAL-MART has been able to successfully become the world's largest retailer, at least part of the credit to its management of the global supply chain network era analysis program. WAL-MART's success is that it not only lead the application of large data analysis, it is more important to apply this analysis to the entire supply chain.

\section{Application Prospect of Big Data Analysis Method}

Data analysis method change a lot of business areas, large data opened the transformation of a great era, in the era of big data, we can more data analysis and sometimes can even all related data processing and a special phenomenon, rather than relying on random sampling, if the enterprises can integrate data from other sources, to obtain more value. These data may come from the suppliers in the supply chain, such as product name, price and discount; also may be goods into the market data, such as certain goods and increase or plans to reduce its yield; may also is the inventory data. These data are crucial for the successful delivery of demand goods in the supply chain.

\section{Conclusion}

Big data will bring benefits to society in many ways. Because big data has become a powerful weapon to solve the pressing global problems and develop the economy. However, the era of big data also put us out of the challenge, we need to be fully prepared to meet the big data technology, our institutions and their own change. Big data marks the human in seeking the way of quantifying and understanding the world made a big step forward, in measurement, storage, analysis and sharing of a lot of things are data, with large amounts of data and more less accurate data for us to understand the world opened up a new door.

\section{Acknowledgements}

This is the social science fund research base project of Beijing-Beijing logistics resources optimization allocation in the coordinated development of Beijing-Tianjin-Hebei research (project number: 15 jdjgb 054) research.

\section{References}

[1] Zhang Yin, Chen Min, Liao Xiaofei. The present situation and Prospect of the application of large data [J]. computer research and development, 2013. 50 (9): 216-233.

[2] Dou Wanchun, Jiang Cheng. The technology system and potential problems of big data applications [J]. ZTE technology, 2013. 19 (4).

[3] Wang Qinmin. Application of big data in economic and social development [N]. Journal of geography, 2015. 70 (5).

[4] Yan Zhixin. Big data on the impact of the retail industry procurement and supply chain management $[\mathrm{J}]$. industrial economy, 2015. 46.

[5] He Jiao. The application prospect of [J]. logistics engineering and management of large data in JIT procurement, 2014. 07 (49).

[6] Zeng Nili. Application of big data in manufacturing enterprise [J]. database and information management, 2014.20 (07).

[7] Ye Bin. Research on the application of big data in logistics enterprise [J]. logistics technology, 2014. 33 (08).

[8] He Rongxuan. The traditional logistics to modern supply chain management transformation strategy $[\mathrm{J}]$. enterprise economy, 2014. 373 (09).

[9] Tian Xue. The application of big data in logistics enterprise [D]. modern service industry.2015.01 (17): 36-37.

[10] Nada R. Sanders. large data supply chain [M]. Beijing: Renmin University of China press, 2015, 31-36.

\section{Biography}

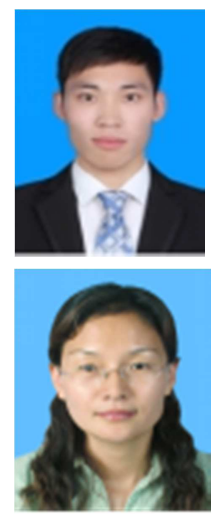

Dou Xin-Xin (1993-), male, hebei province, graduate student of Beijing Wuzi University, the main research direction for the logistics integration of the Beijing-Tianjin-Hebei region, the logistics park planning.

Wang Xiao-ping (1977-), female, shandong province, Associate professor and Associate Dean, of School of logistics, Beijing Wuzi University, $\mathrm{Ph}$. D., main research direction for the logistics integration of the Beijing-Tianjin-Hebei region, logistics information, etc. 